\title{
Development of a Human Head FE Model and Impact Simulation on the Focal Brain Injury*
}

\author{
Dai WATANABE**, Kohei YUGE**, Tetsuya NISHIMOTO***, \\ Shigeyuki MURAKAMI**** and Hiroyuki TAKAO***** \\ ** Seikei Univ. Faculty of Science and Technology \\ 3-3-1, Kitamachi, Kichijoji, Musashino-shi, Tokyo, Japan \\ E-mail: watanabe@cml.me.seikei.ac.jp : yuge@st.seikei.ac.jp \\ *** Nihon Univ. College of Engineering \\ 1, Tokusada-Nakagawa, Tamura-machi,Koriyama-shi, Fukushima, Japan \\ E-mail: tnishi@mech.ce.nihon-u.ac.jp \\ **** Murakami Clinic \\ 7-38-8, Ichinoe, Edogawa, Tokyo, Japan \\ E-mail: fundan@mac.com \\ ***** Jikei Univ. School of Medicine \\ 3-25-8, Nishishinbashi, Minato, Tokyo, Japan \\ E-mail: takao@jikei.ac.jp
}

\begin{abstract}
In this paper, a three-dimensional digital human-head model was developed and several dynamic analyses on the head trauma were conducted. This model was built up by the VOXEL approach using 433 slice CT images $(512 \times 512$ pixels $)$ and made of 1.22 million parallelepiped finite elements with 10 anatomical tissue properties such as scalp, CSF, skull, brain, dura mater and so on. The numerical analyses were conducted using a finite element code the authors have developed. The main features of the code are 1) it is based on the explicit time integration method and 2) it uses the one point integration method to evaluate the equivalent nodal forces with the hourglass control proposed by Flanagan and Belytschko ${ }^{(1)}$ and 3) it utilizes the parallel computation system based on MPI. In order to verify the developed model, the head impact experiment for a cadaver by Nahum et al. ${ }^{(2)}$ was simulated. The calculated results showed good agreement with the experimental ones. A front and rear impact analyses were also performed to discuss on the characteristic measure of the brain injury, in which the von-Mises stress was high in the frontal lobe in both of the analyses because of the large deformations of a frontal cranial base. This result suggests that the von-Mises stress can be a good measure of the brain injury since it is empirically well known that the frontal lobe tends to get injured regardless of the impact positions.
\end{abstract}

Key words: Biomechanics, Computational Mechanics, Finite Element Analysis, VOXEL Approach, Head Trauma, Focal Brain Injury

*Received 26 Dec., 2008 (No. T1-07-0741) Japanese Original : Trans. Jpn. Soc. Mech. Eng., Vol.74, No.740, A (2008), pp.611-620 (Received 17 Aug., 2007) [DOI: $10.1299 /$ /cst.3.252]

\section{Introduction}

A human brain is the most important organ for life activity and humanity and is also a vulnerable organ which tends to have residual disabilities by vehicle accidents because neuron cells don't repair themselves basically. Therefore, it is very important for auto mobile safeties to protect a brain from severe injuries. However, the brain injury mechanism due to impact loads has not been made clear because of the limitations of the experimental 
data. For example, in focal brain injuries, a brain injury frequently occurs at an opposite area of the impact position, which is called the contrecoup injury, but it hasn't been made clear why such injuries occur. Several hypotheses have been proposed for this contrecoup injury, including the cavitation theory by $\operatorname{Gross}^{(3)}$ and the shear strain theory by Holbourn ${ }^{(4)}$, but none of them has been proven.

Some research groups have studied the brain injury mechanism using human surrogates $^{(5)(6)}$ and others using physical human head models ${ }^{(7)(8)}$. Recent development of computers has enabled computer simulations of a human head subjected to impact loads. 2-D simple head models were made to understand the response of a brain to an impact by Ruan et al. ${ }^{(9)}$ Nishimoto, one of the present authors, et al. ${ }^{(10)(11)}$ used 2-D coronal and sagittal plane head model to simulate a brain behavior subjected to the rotational impact. Zhou et al. ${ }^{(12)}$ made a 3-D head FE model, with which they performed impact simulations and suggested that focal injuries are induced by pressure while diffuse axonal injuries are induced by shear stresses. Kleiven et al. ${ }^{(13)}$ reported the influence of the head size on head injuries. Zhang et al. ${ }^{(14)}$ developed one of the most detailed head models called WSUBIM version 2001. In spite of these studies the mechanisms of head injuries has not been clarified yet.

In this research a precise human head FE model was developed by the VOXEL approach to study the relationship between the impact loads and the head responses. Then the model was validated by simulating an existing cadaver impact experiment and comparing the dynamic responses. Furthermore several simulations were performed to study the relation between the impact direction and the head injuries.

\section{Construction of a head FE model}

\subsection{VOXEL modeling}

The VOXEL method proposed by Hollister et al. ${ }^{(15)}$ was used for the head modeling. This method construct a 3-D FE model easily by laminating the cross sectional images such as CT pictures with appropriate thickness. The main benefit of employing this method is that a 3-D FE model can be made almost automatically if only we have the sectional images of the object. Moreover, the method can automatically construct the detail geometry of a human head which has a complicated asymmetrical shape and tiny projection on a skull. The weak point of the method is that the method requires a lot of finite elements. But recent development of computer is changing it a smaller issue.

\subsection{Sectional Images Acquisition}

High resolution CT images of a male volunteer in his twentieth were obtained. The resolution was $0.4375[\mathrm{~mm}] \times 0.4375[\mathrm{~mm}]$ and the slice pitch was $0.6[\mathrm{~mm}]$. The number of the images was 433 .

\subsection{Classification of Tissues and Image Processing}

It is necessary to get a clear CT image that the densities of the target constituents are relatively different. A human head, however, is composed of many tissues having very similar densities, so it is difficult to classify all the constitutive tissues. Thus, the head was classified into 10 tissues (skin, soft tissue, CSF, eye, skull, brain, falx cerebri, dura mater, tentorium cerebelli, brain ventricle) and clear bitmap images were made with them to construct the VOXEL head model. The white matter of the brain was reported to be stiffer than gray matter in Ref.(14) whereas the gray one was stiffer in Ref.(16). We assumed that they have same mechanical properties in this study.

A pixel data of a CT slice image saved by the DICOM (Digital Imaging and Communications in Medicine) format has a 12[bit] CT value data besides a 256 gray scale image data. Thus, from the same CT slice image, the skin and skull were classified using a 
wide range contour mode of a CT viewer (Fig. 1(a)) and the others like a brain, soft tissue and ventricle were done using a narrow contour mode (Fig. 1(b)). Then the bitmap image classified into 10 tissues was composed by superimposing their pictures (Fig. 1(c)).
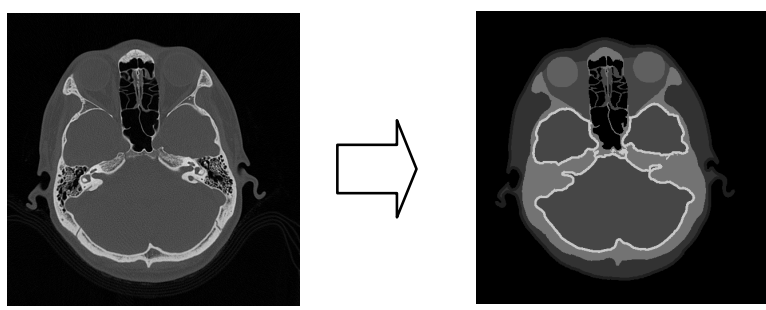

(a) Classified tissues from linear mode image
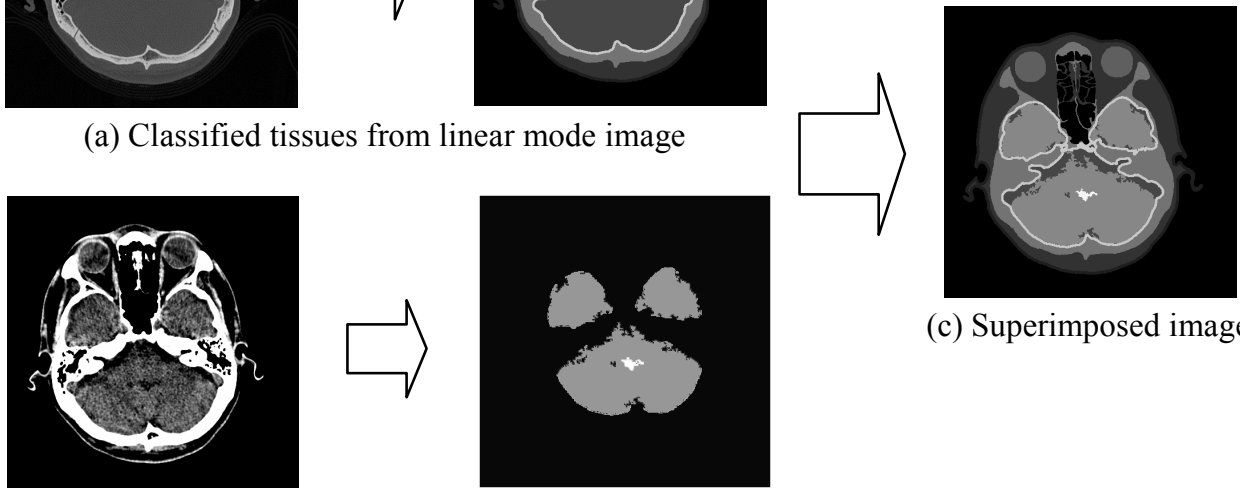

(c) Superimposed image

(b) Classified tissues from brain mode image

Fig. 1 Image processing procedure

\subsection{3-D Model Construction}

Based on the bitmap images using the aforementioned procedure, 3-D human head model was developed. Several tissues of the model are shown in Fig. 2 and a complicated shape in a frontal cranial base is shown in Fig. 3. As can be seen from Fig. 2 and Fig. 3, geometry of these tissues is modeled in details.

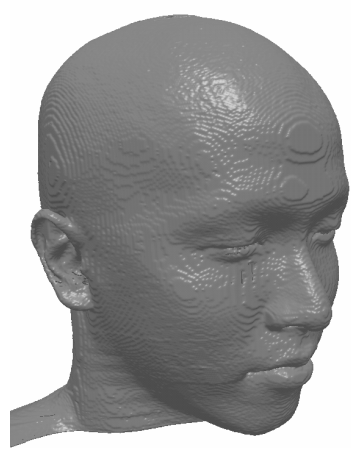

(a) Skin

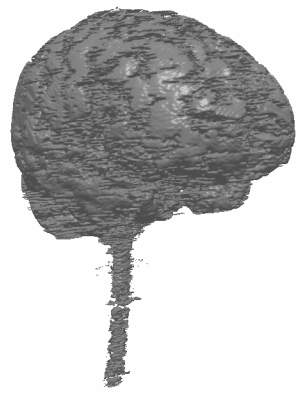

(d) Brain

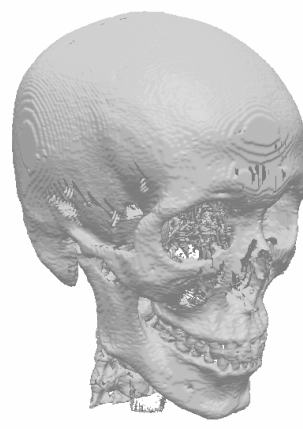

(b) Skull

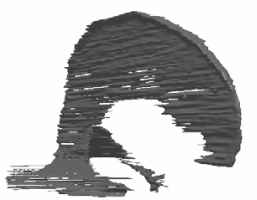

(e) Falx cerebri and

Tentorium cerebelli

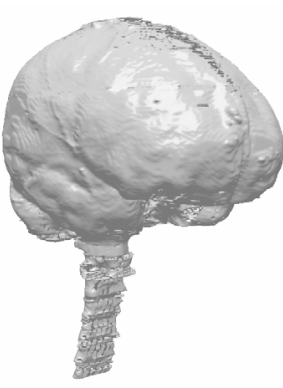

(c) Dura mater

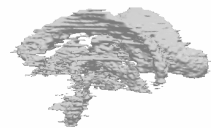

Fig. 2 Tissue models of a human head (40million elements model) 
This original model is composed of about 40 million hexahedron elements. The author, however, utilized a simplified model to adjust to his computational environment to make the computer calculation more practical. The simplified model has 1.22 million elements by compressing the bitmap sectional image from $512 \times 512$ [pixel] to $128 \times 128$ [pixel], and has 216 layers by reducing the number from 433 to 216 . As shown in Fig. 4, the simplified model maintains precision of the original model in details. The simplified model was used in the following numerical analyses.

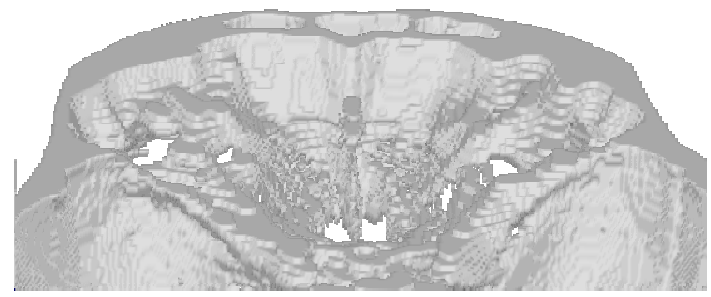

Anterior

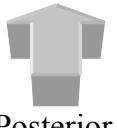

Posterior

Fig. 3 Detailed frontal cranial base modeling

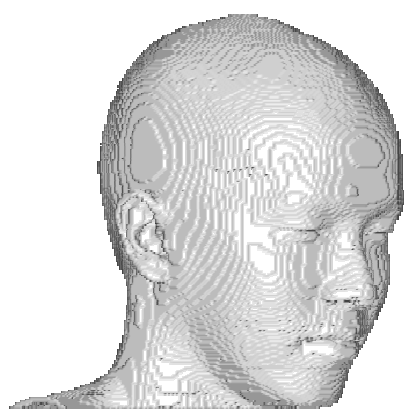

(a) Skin

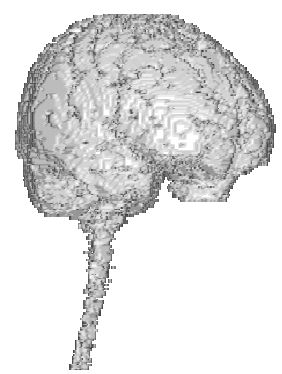

(d) Brain

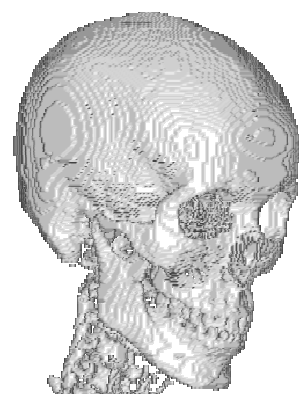

(b) Skull

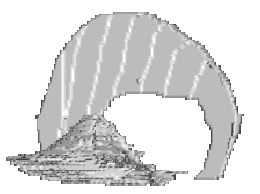

(e) Falx cerebri and

Tentorium cerebelli

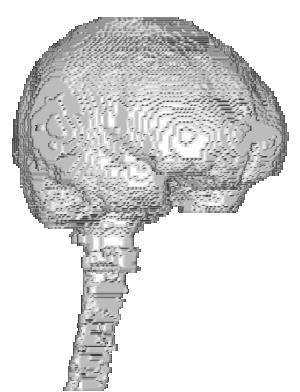

(c) Dura mater

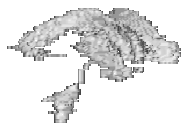

(f) Ventricle

Fig. 4 1.22million elements Human head model

\section{Model specifications and validation}

\subsection{Computational environment}

A FEM code based on the explicit time integration method was developed for impact simulations of the VOXEL head model. It uses the one point integration method to evaluate the equivalent nodal forces with the hourglass control proposed by Flanagan and Belytschko ${ }^{(1)}$. The Updated Lagrangian formulation was used for large deformations. In addition, a parallel computation algorithm with MPI (Message Passing Interface), which enables low-cost parallel computation, was adopted. A PC cluster composed of 8 PCs (CPU:Athlon64 3000+) was used in the present analyses. The acceleration ratio of parallel computation in the head impact analyses was about 6.5. It took about 7.5 hours for the PC cluster to simulate the impact behaviors of the human head for $8[\mathrm{~ms}]$ duration, the details of which is described in Section 3.3. 


\subsection{Material properties}

A head subjected to an impact load moves translationally and rotationally. In that time, relative motions of the brain surface and the cranial inside are expected. So it is more important how to treat the cerebral spinal fluid (CSF) which fills in a cranial space. The sliding interface, or the ALE(Arbitrary Lagrangian and Eulerian) method is often employed for the fluid-solid coupling problems, but these methods may not be useful in the present VOXEL head model because it uses rectangular solid elements and doesn't have a smooth interface between the brain and the CSF. So we assumed that the CSF doesn't show fluid behaviors in impact duration (about $10[\mathrm{~ms}]$ ) and treated the CSF with the Generalized Maxwell visco-elastic model as shown in Fig. 5.

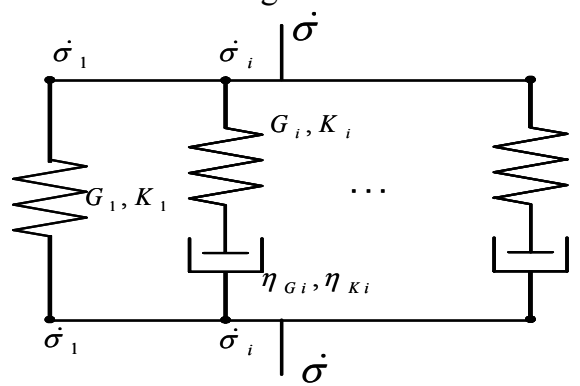

Fig. 5 Generalized Maxwell visco-elastic model

The generalized Maxwell visco-elastic model for the 3D stress field is expressed as

$\{\dot{\sigma}\}=\left\{\dot{\sigma}_{1}\right\}+\sum_{i}\left\{\dot{\sigma}_{i}\right\}=\left(\left[D_{1}\right]+\sum_{i}\left[D_{i}\right]\right)\{\dot{\varepsilon}\}-\sum_{i}\left(\frac{1}{T_{G i}}\left\{\sigma_{i}^{\prime}\right\}+\frac{1}{T_{K i}}\left\{\sigma_{m i}\right\}\right)$

Where $\{\dot{\sigma}\}$ and $\{\dot{\varepsilon}\}$ are the stress and strain velocities respectively, $\left\{\sigma^{\prime}\right\}$ and $\left\{\sigma_{m}\right\}$ express the deviatororic stresses and the hydrostatic pressure. The index $i$ is the component number of the generalized Maxwell visco-elastic model. $T_{G i}$ and $T_{K i}$ represent the stress relaxation times and defined as $T_{G i}=\eta_{G i} / G_{i}$ and $T_{K i}=\eta_{K i} / K_{i}$ where $G_{i}$ is the shear modulus, $K_{i}$ is the bulk modulus, and $\eta_{G i}, \eta_{K i}$ are the viscosity coefficients. In the present analyses a single spring dashpot model with $K=2.19 \times 10^{9}[\mathrm{~Pa}], \quad G=500[\mathrm{~Pa}]$ and $\eta=1.0 \times 10^{-3}[\mathrm{~Pa} \cdot \mathrm{s}]$ was used, assuming that the CSF is nearly incompressible and has a very low viscosity. $T_{G}$ is calculated as $2.0 \times 10^{-6}[\mathrm{~s}]$ with these values.

In order to verify the CSF material properties, a drop test of a spherical shell filled with water and the FE analysis of water using the above Maxwell model was conducted. The shell is made of acrylic resin. The outer and inner diameters of the shell are $150[\mathrm{~mm}]$ and $145[\mathrm{~mm}]$. An acceleration sensor and two pressure sensors were attached at an angle of 45-degree from the top and bottom of the sphere respectively as shown in Fig. 6(a). The rig was dropped from a height of $200[\mathrm{~mm}]$ to a buffer(Fig. 6(b)).

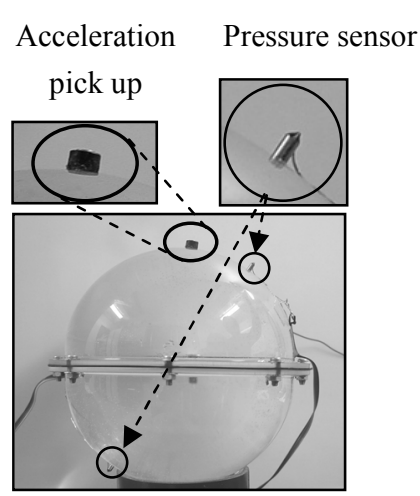

(a) Test rig

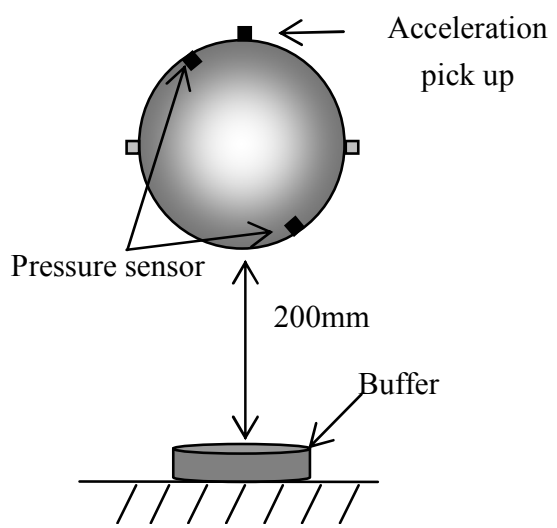

(b) Schematic of experiment

Fig.6 Free-fall impact experiment for studying pressure response 
The time histories of the pressure obtained by the numerical analysis agree well with the experimental ones as shown in Fig. 7. This comparison suggests that the present Maxwell model for the water wouldn't cause a major problem to treat the CSF.

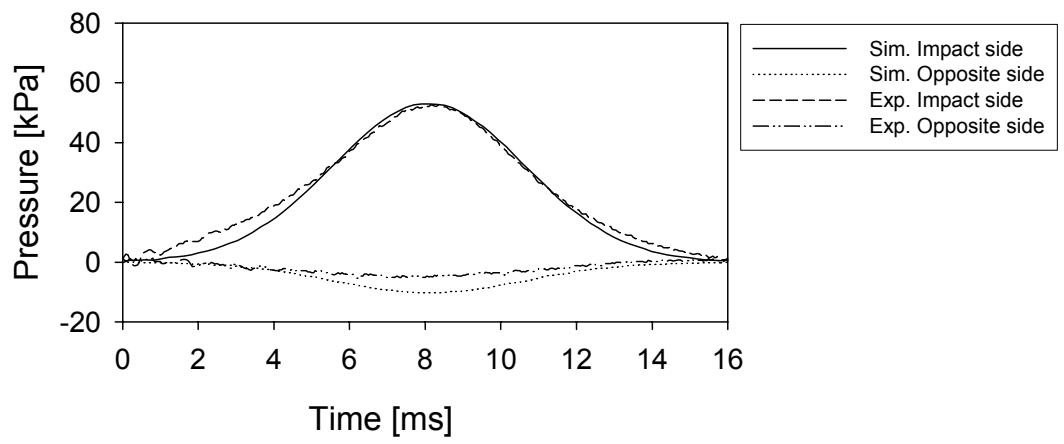

Fig. 7 Time history of simulation pressure compared with the experimental pressure

Several elastic and visco-elastic material properties were proposed for a brain by Sauren et al. ${ }^{(17)}$ The visco-elastic models proposed by them are expressed using 3-elements Maxwell model as shown in Eqs. (2).

$$
G(t)=G_{\infty}+\left(G_{0}-G_{\infty}\right) e^{-\beta t}
$$

Where $G_{0}, G_{\infty}, \beta$ and $t$ show a short term shear modulus, long term shear modulus, decay factor and duration respectively. $G_{0}, G_{\infty}$ and $\beta$ are also corresponding to $G_{1}+G_{i}, G_{1}$ and $1 / T$ respectively in Fig. 5. In this study, the skull was assumed to be elasto-plastic and the material properties proposed by Nishimoto et al. ${ }^{(18)}$ were used. The skin and the membrane were treated as an elastic material with the material properties proposed by Ruan et al. ${ }^{(19)}$ The brain was assumed to be the 3-elements Maxwell visco-elastic material model proposed by Zhang et al. The material properties of each tissue used for the head model are shown in Table 1.

Table 1 material properties of tissues in the head model

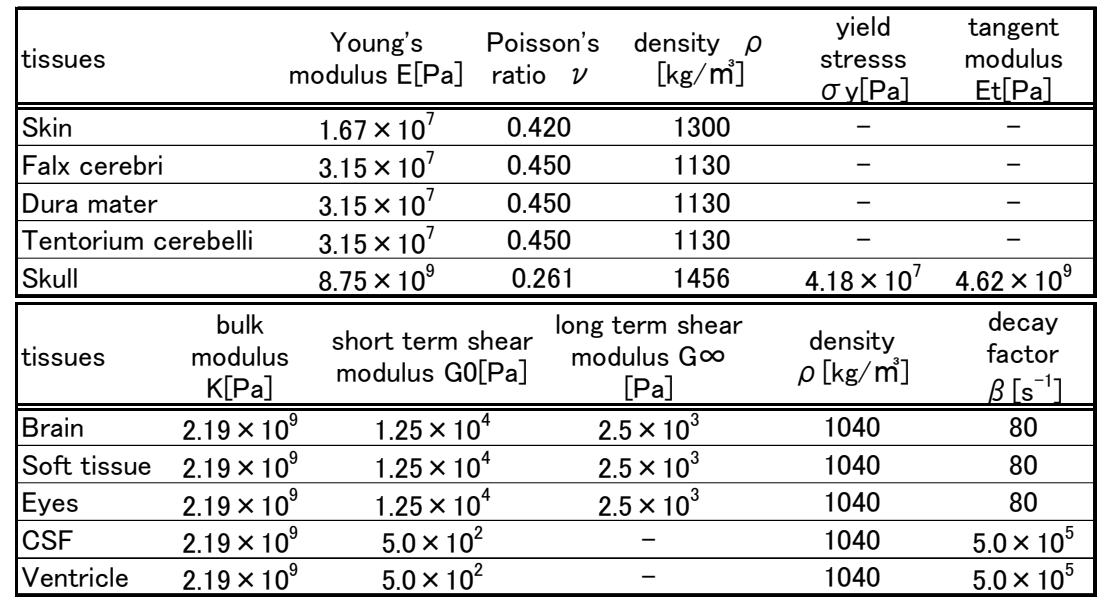

\subsection{Validation of the Model}

One of the well-known experimental studies related to an impact response of a human head is the one by Nahum et al. ${ }^{(2)}$, in which a $5.59[\mathrm{~kg}]$ impactor was hit horizontally at the speed of $9.94[\mathrm{~km} / \mathrm{h}]$ on the forehead of a cadaver and pressure histories in several points of an intracranial area were measured. To verify the developed FE head model this experiment was simulated. The calculated pressure distribution in a sagittal plane at $2.5[\mathrm{~ms}]$ is shown in Fig. 8 and the pressure histories of the points(Fig. 9) were compared with the experimental ones in Fig. 10. The difference of the calculated and the experimental pressure was no more 
than $10 \%$ at most. Considering the individual differences of a head shape, size and weight, it can be said that the calculated curves are very close to the experimental ones. This result suggests that the developed model is useful for head injury simulations.

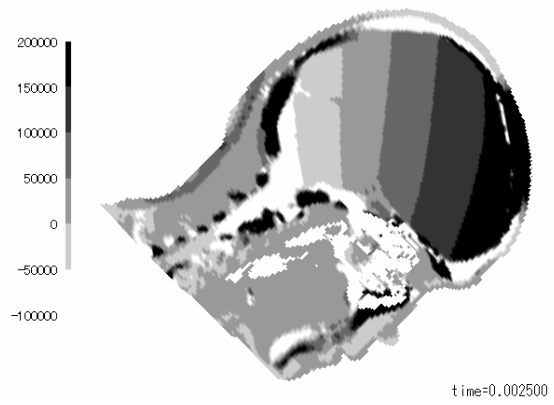

Fig. 8 Pressure distribution in the sagittal plane at $2.5[\mathrm{~ms}][\mathrm{Pa}]$

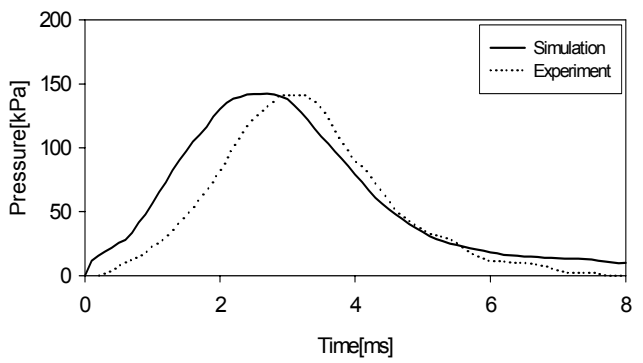

(a) Frontal

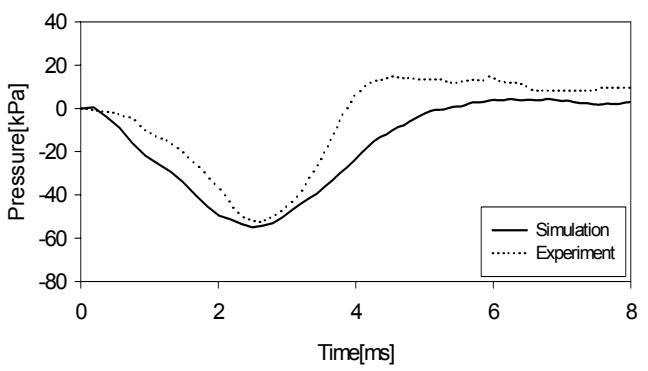

(c) Occipital

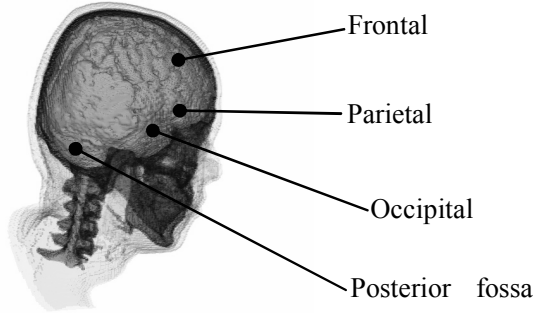

Fig. 9 Location of the pressure measurement

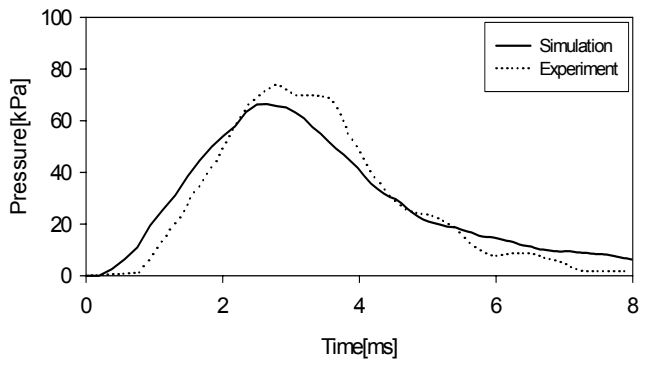

(b) Parietal

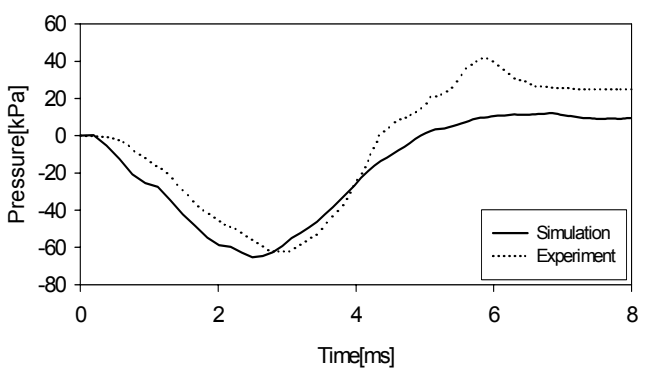

(d) Posterior fossa

Fig. 10 Time history of intracranial pressure compared with Nahum's experiment

\section{Simulation for an evaluation of a head trauma}

\subsection{Frontal and occipital head impact simulation}

Sano et al. ${ }^{(20)}$ investigated the relationship between impact directions and injury areas in a head by many autopsy reports. In their report, coup injuries were observed in $80 \%$ of the cases where frontal areas were impacted while contrecoup injuries were observed in $80 \%$ of the cases where occipital areas were impacted. The report indicates that the injury is more likely to occur in a frontal area than in an occipital area regardless of impact directions. Although the research result is widely known, the reason has not been clarified yet. In order to study the focal injury mechanism, the frontal and occipital head impact simulations were conducted. The load was a sinusoidal wave with $10[\mathrm{~ms}]$ duration and $8000[\mathrm{~N}]$ at its peak. The load was applied to the center of a forehead (the frontal impact analysis) and to the back head (the occipital impact analysis) as shown in Fig. 11. 


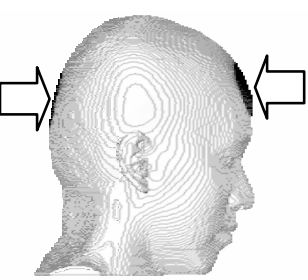

(a) Side view

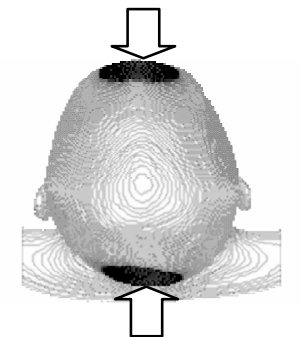

(b) Top view

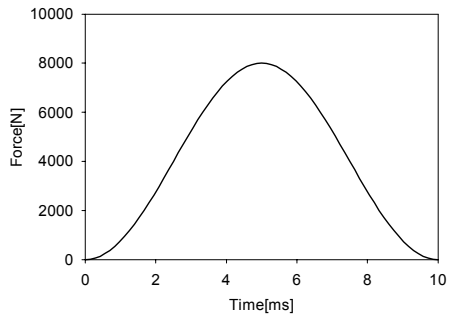

(c) Applied force-duration curve

Fig. 11 Load points and Load-duration curve applied in a frontal and occipital impact

\subsection{The result of the simulation}

The pressure distributions in a horizontal plane at the maximum load are shown in Fig. 12. The pressure changes linearly from the impact side to the opposite side in both analyses. The maximum absolute value of the pressure is much higher in the impact side than in the opposite side. These results imply that the pressure is not a good indicator to demonstrate the clinical knowledge that the anterior part of the brain tends to be injured regardless of the impact position. On the other hand, the von-Mises stress becomes high in the bottom of the frontal lobe in both analyses as shown in Fig. 13. The von-Mises stress is often used in the yield conditions of metals and is defined using the principal stresses $\sigma_{i}(i=1,2,3)$ as follows,

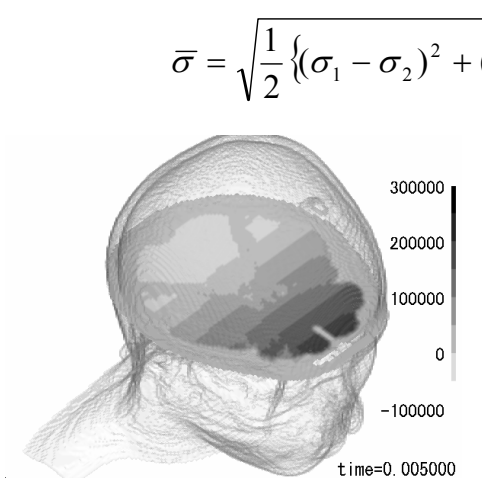

(a) Front impact (a) Front impact

Fig. 12 Pressure distribution under an impact load $[\mathrm{Pa}](\mathrm{t}=5 \mathrm{~ms})$

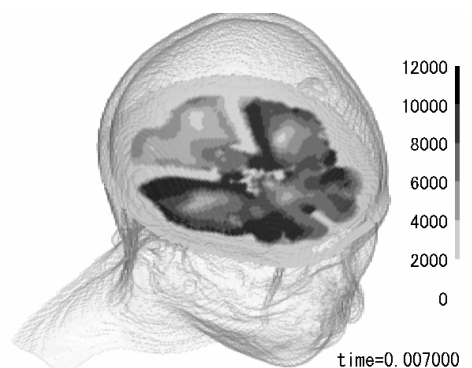

(a) Front impact

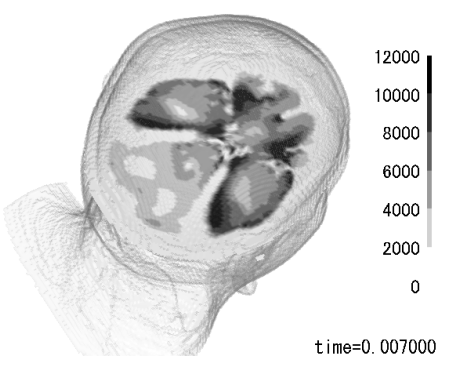

(b) Rear impact

Fig. 13 von-Mises stress distribution under an impact load $[\mathrm{Pa}](\mathrm{t}=7 \mathrm{~ms})$

\section{Discussion}

It is observed that the pressures change linearly from the impact side to the opposite side in both analyses and the absolute value of pressure is higher in the impact side than the opposite side. According to Ruan et al. ${ }^{(19)}$, this was caused by compressive deformations and acceleration of the head. That is to say, compressive deformations by the impact load, in most cases, produce positive pressure in the brain. As a result, the pressure is linearly distributed as shown in Fig. 12.

In the cavitation theory by Gross, a brain injury occurs in the occipital by the frontal impact, which, however, contradicts with the report by Sano et al. that the head injury tends 
to occur in a frontal area regardless of impact directions. Meanwhile the von-Mises stress was high near the bottom of the frontal lobe, in both the frontal and occipital impact analyses. Fig. 14 shows a clinical example of contrecoup injury, where the swelling of a skin and the bone fracture are found at the occipital impact point(Fig. 14(a)) and the contusion is found at the bottom of a frontal lobe (Fig. 14(b)). The contusion position is close to the calculated high stress area in Fig. 13(b).

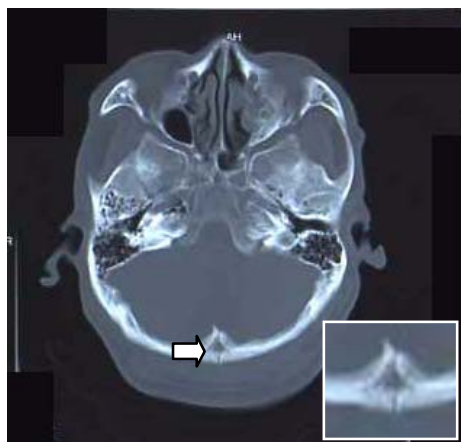

(a) Bone fracture and swelling of skin

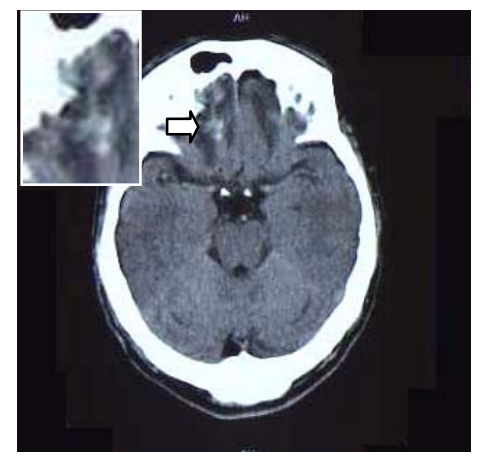

(b) Contusion

Fig. 14 CT image of contrecoup contusion in an occipital impact

In order to study the mechanism of higher stresses distribution in a frontal lobe, especially the bottom of a frontal lobe, the time history of pressures and von-Mises stresses at the specific points in the near impact and the bottom of a frontal lobe(Fig. 15) are shown in Fig. 16 and Fig. 17 respectively. It can be seen that the pressure marked maximum at the maximum load while the von-Mises stress marked maximum after the maximum load.

The main reason why higher stresses occur in the bottom can be attributed to the deformation of the thin wall structure of the frontal base of skull caused by the head acceleration, rather than the head deformation by the impact load. In both analyses a frontal base of skull was deformed largely as shown in Fig. 18 and Fig. 19. The thickness of this base is approximately $0.1[\mathrm{~mm}]$ and is quite deformable. Therefore, it is highly likely to say that the sudden move of a head by an impact load induces large deformations of a frontal base of skull and subsequently, the deformations yield high stresses beyond the injury tolerance in the bottom of frontal lobe. Based on this hypothesis, we can explain the clinical tendency that the injury frequently occurs in a frontal area regardless of impact directions.

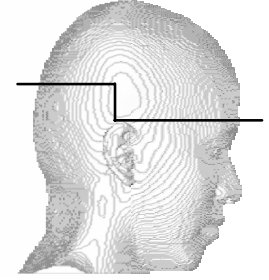

(a) cut section

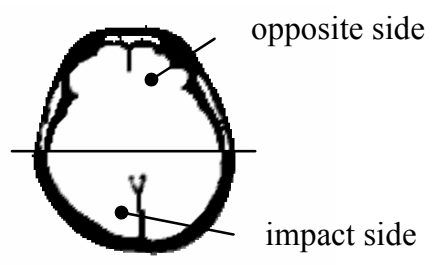

(b) horizontal plane

Fig. 15 Location of the measurement points

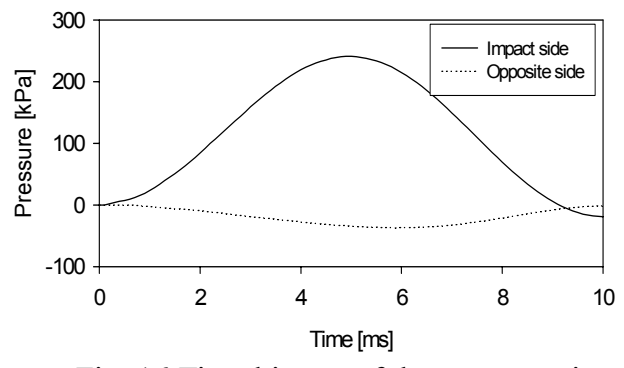

Fig. 16 Time history of the pressures in the rear impact

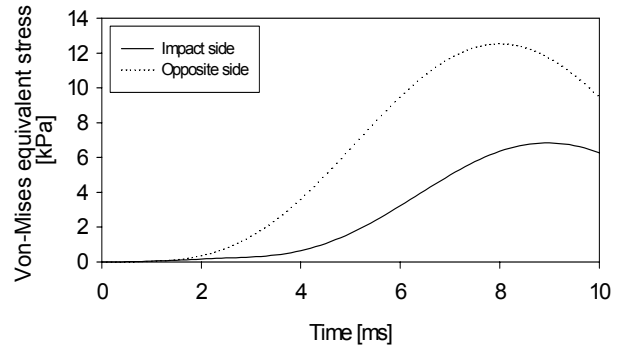

Fig. 17 Time history of the Mises equivalent stresses in the rear impact 


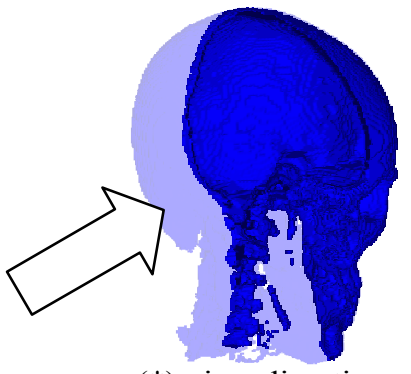

(*) view direction

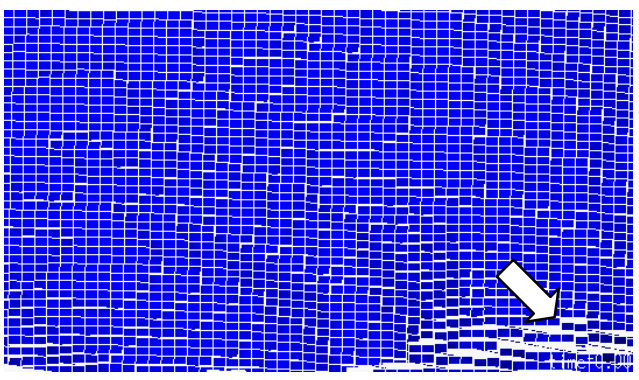

(a) $0.0[\mathrm{~ms}]$

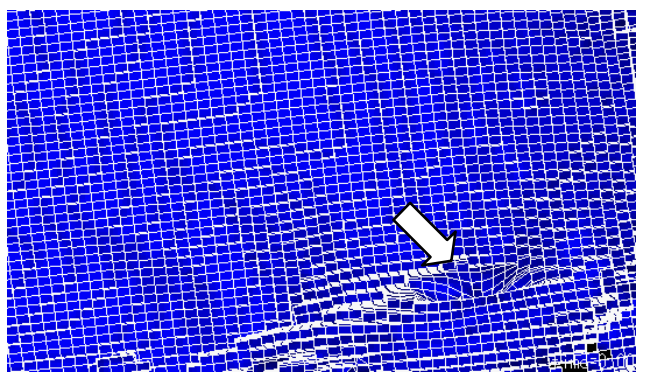

(c) $4.0[\mathrm{~ms}]$

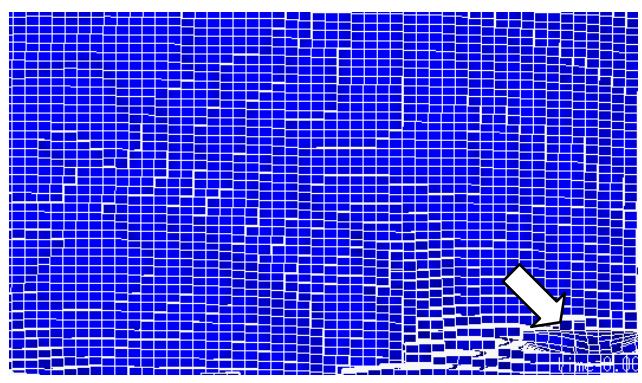

(b) $2.0[\mathrm{~ms}]$

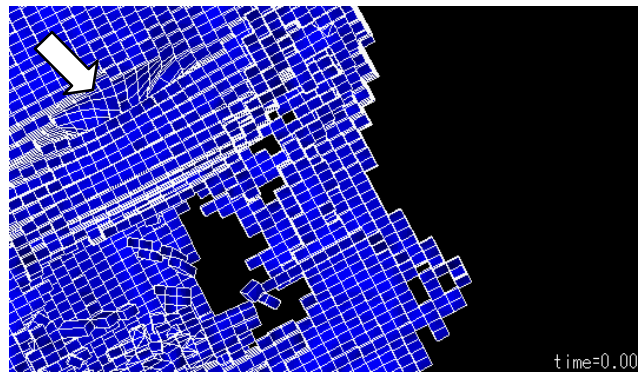

(d) $6.0[\mathrm{~ms}]$

Fig. 18 a frontal base of skull deformed largely at arrow points while a frontal impact (shown with 15 times large displacement of actual)

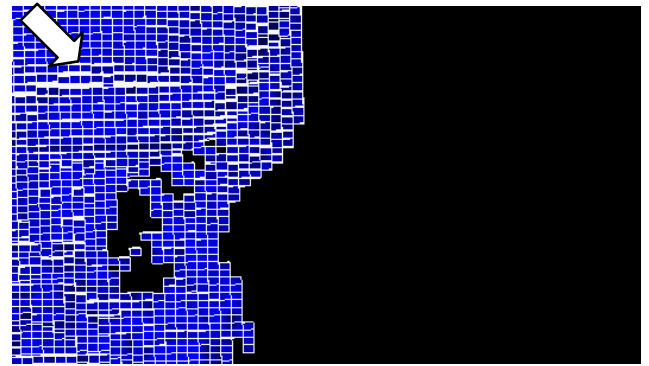

(a) $0.0[\mathrm{~ms}]$

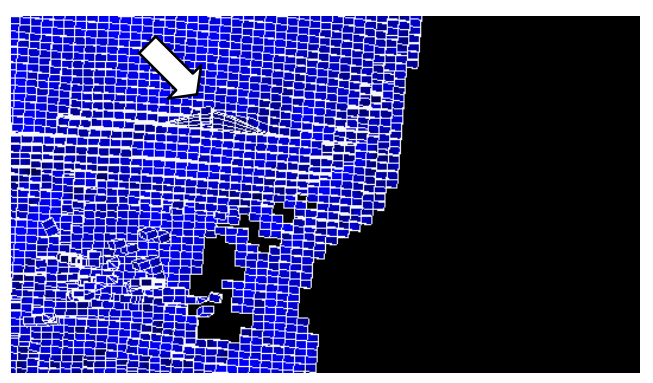

(c) $4.0[\mathrm{~ms}]$

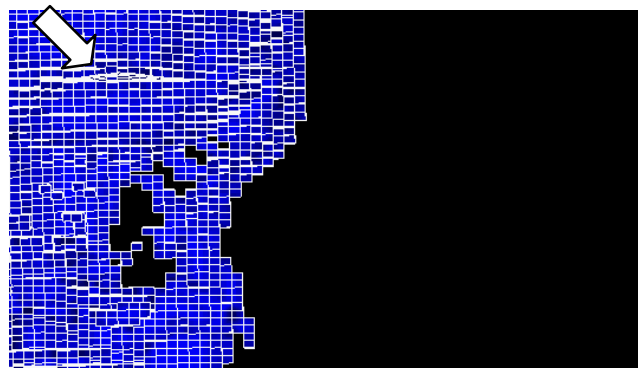

(b) $2.0[\mathrm{~ms}]$

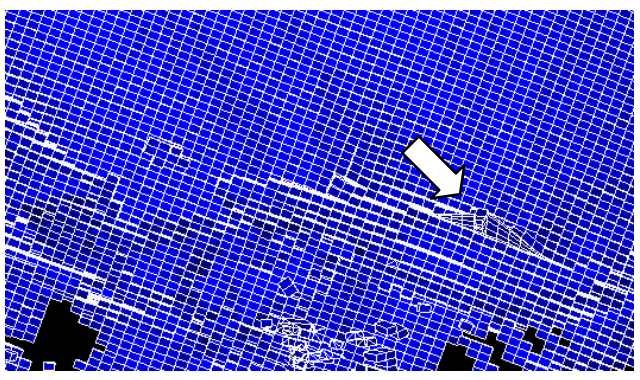

(d) $6.0[\mathrm{~ms}]$

Fig. 19 a frontal base of skull deformed largely while an occipital impact (shown with 15 times large displacement of actual) 
However, the head injuries would not occur by a sole mechanism. There is an experimental report that the incubated nerve cells were damaged by negative pressure ${ }^{(21)}$. Therefore, further numerical studies under various load conditions are necessary to understand the head injury mechanism. In addition, the material properties proposed in various reports were employed in this research. The verification of them through numerical analyses remains to be solved.

\section{Conclusions}

In this research, a precise human head FE model based on the VOXEL approach was developed to study the head injury mechanism. The verification of the model was conducted by simulating the cadaver test reported by Nahum et al. The calculated pressure history curves in the brain agreed well with the experimental ones. A frontal and occipital impact analyses were also performed to investigate the relation between the impact direction and the positions of the high measurement of pressure and stresses in brain. The obtained results represent that brain injury has a closer relation with the Mises equivalent stress than the pressure. At this time, the large deformation of a frontal cranial base was observed in both frontal and occipital impact analyses. We proposed a new hypothesis that the sudden move of a head by an impact load induces the deformation of a frontal base of skull and the deformation induces injuries in the bottom of frontal lobe subjected to a stress over its tolerance for the focal injury mechanism that the injury frequently occurs in a frontal area regardless of impact directions.

\section{Acknowledgment}

"High-Tech Research Center" Project for Private Universities : matching fund subsidy from MEXT (Ministry of Education, Culture, Sports, Science and Technology), 2008.

\section{References}

(1) Flanagan, P. D., and Belytschko, T., A Uniform Strain Hexahedron and Quadrilateral with Orthogonal Hourglass Control, International journal for numerical methods in engineering, Vol. 17, pp. 679-706

(2) Nahum, A.M. et al., Intracranial Pressure Dynamics During Head Impact, Proc. 21st Stapp Car Crash Conf., SAE Paper, No.770922(1977), pp. 339-366

(3) Gross, R.H., A new theory on the dynamics of brain concussion and brain injury, $J$ Neurosurg., Vol. 15(1958), pp. 548-561

(4) Holbourn, A.H.S., Mechanics of head injuries, Lancet, Vol. 2(1943), pp. 438-441

(5) Ommaya, A.K. and Hirsch, A.E., Tolerances for Cerebral Concussion from Head Impact and Whiplash in Primates, J. Biomechanics, Vol. 4(1971), pp. 13-21

(6) Gennarelli, T. A. et al., Diffuse Axonal Imjury and Traumatic Coma in the Primate, Annals of Neurology, Vol. 12(1982), No.6, pp. 564-574

(7) Prnaik, Y. et al., The Influence of Surrogate Blood Vessels on the Impact Response of a Physical Model of the Brain, Stapp Car Crash Journal, Vol. 48(2004), pp. 1-19

(8) Bradshaw, D.R.S. et al., Simulation of acute subdural hematoma and diffuse axonal injury in coronal head impact, J. Biomechanics, Vol. 34(2001), pp. 85-94

(9) Ruan, J.S. et al., Human Head Dynamic Response to Side Impact by Finite Element Modeling, ASME J. Biomechanical Eng., Vol. 113(1991), pp. 276-283

(10) Nishimoto, T. et al., Stress Distribution of Brain under Rotational Impact, Trans. of JSME, Series A, Vol. 61, No. 601(1996), pp. 2109-2115

(11) Nishimoto, T. and Murakami, S., Correlation between Disturbance of Consciousness and Impact Stress Concentration of Brain, Trans. of JSME, Series C, Vol. 63(1997), pp. 715-721 
(12) Zhou, C. et al., A new model comparing impact responses of the homogeneous and inhomogeneous human brain, Proc.39th Stapp Car Crash Conference, SAE Paper No.952714(1995), pp. 121-137

(13) Kleiven, S. and Holst, H. v., Consequences of head size following trauma to the human head, J. Biomechanics, Vol. 35(2002), pp. 153-160

(14) Zhang, L.et al., Recent Advances in Brain Injury Research: A New Human Head Model Development and Validation, Stapp Car Crash Journal, Vol. 45(2001), pp. 369-394

(15) Hollister, J.S., and Kikuchi, N., Homogenization Theory and Digital Imaging: A Basis for Studying the Mechanics and Design Principles of Bone Tissue, Biotechnology and Bioengineering, Vol. 43 (1994), pp. 586-596

(16) Nicolle, S., at al., Shear Properties of Brain Tissue over a Frequency Range Relevant for Automotive Impact Situations: New Experimental Results, Stapp Car Crash Journal, Vol. 48(2004), pp. 1-20

(17) Sauren, A.A.H.J. and Claessens, M.H.A., Finite Element Modeling of Head Impact: The Second Decade, Proc. Int. IRCOBI Conference (1993), pp. 241-254.

(18) Nishimoto, T. et al., Mechanical Properties of Human Cranium and Effect of Cranial Fractures on Extradural Hematoma, Trans. of JSME, Series A, Vol. 61, No. 591(1995), pp. 2386-2392

(19) Ruan, J.S.and Prasad, P., Study of the Biodynamic Characteristics of the Human Head, International Research Council on the Biomechanics of Impact(1996), pp. 63-73

(20) Sano, K. et al., Mechanism and dynamics of closed head injuries, Neurol Medicochir., Vol. 9(1967), pp. 21-33

(21) Nakamachi, E. et al., Development of a Stress Standard Base Criterion for Nerve Cell Injury by Hydrostatic Pressure Loading, Trans. of JSME, Series A, Vol. 73, No. 734(2007), pp. $1183-1188$ 